\title{
A Discrete PID Control System Using Predictors and an Observer for the Influence of a Time Delay
}

\author{
Y. Hikichi, K. Sasaki, R. Tanaka, H. Shibasaki, K. Kawaguchi, and Y. Ishida
}

\begin{abstract}
In this paper, a control system for the influence of a time delay compensation using predictors and a disturbance observer is proposed. The time delay in the controlled plant is one of the serious problems decreasing the control stability. Therefore, many control methods for controlled plants with a time delay have been proposed until now. We proposed an effective control system for influence of time delay by connecting predictors and a disturbance observer to a PID control system. It alleviates the influence of the time delay on the target response and disturbance response.
\end{abstract}

Index Terms-PID Control, time delay, predictor, disturbance observer.

\section{INTRODUCTION}

PID control is one of the most common control structures employed in the process industry. Therefore, many tuning methods for PID control have been studied and proposed. But PID tuning for controlled plants with a time delay is difficult.

There are several effective control systems for controlled plants with a time delay. For example, Internal Model Control (IMC) [1] and the Smith predictor [2]. However, for plants with integrators, both IMC and the Smith predictor induce a steady state error due to an input-side disturbance; this is serious problem. The approaches used to solve this problem include the modified IMC [3], the modified Smith predictor that use a predictor of the controlled plant [4][5], the method of connecting a PID controller predictor and a model of the controlled plant to the conventional PID control system (PID control with time delay compensation).[6] These are effective methods for reducing the influence of the time delay on the disturbance response. Among them, by using PID control with time delay compensation, while the disturbance response has no errors, it is hardly satisfactory result.

We believe that to resolve this problem, prediction of the disturbance is needed. Therefore, we propose a method to connect predictor of the controller, a discrete observer, and predictors of the controlled plant to the PID control system.

\section{INTERNAL MODEL CONTROL(IMC)}

In Fig. $1, Q(z)$ is the IMC controller, $G_{P}(z)$ is the proper controlled plant model, and $G_{M}(z)$ is measured controlled plant model. IMC feeds back the difference between output of the $G_{P}(z)$ and output of the $G_{M}(z)$. This method is for the

Manuscript received September 31, 2012; revised December 11, 2012

The authors are with School of Science and Technology, Meiji University, Kawasaki-shi, Kanagawa, 2148-571 Japan. (e-mail:ce11075@meiji.ac.jp). amount of operation to re-determine when there is a disturbance as well as modeling errors.

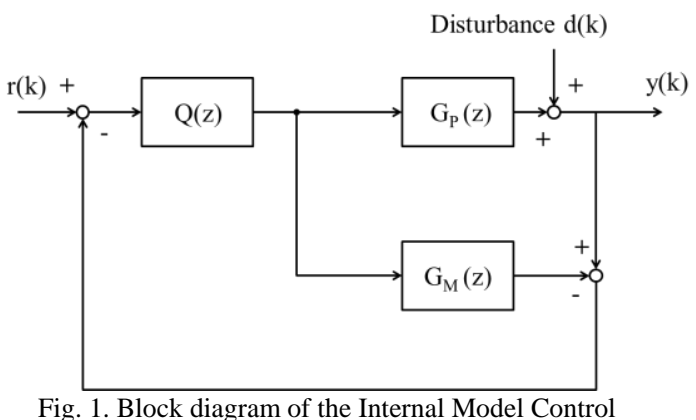

$$
C(z)=F(z) G_{M}(z)^{-1}
$$

In this equation, $F(z)$ is called filter. this means that the degrees of numerator are two degrees less than those of denominator.

\section{Methodology}

\section{A. PID Control.}

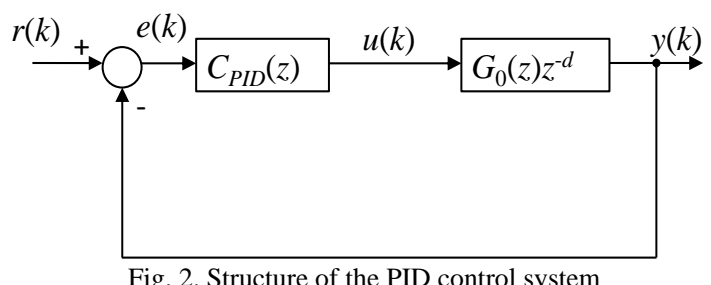

Fig. 2 shows a conventional PID control system. In cases where the controlled plant has a time delay, it is apparent that the control stability decays and PID tuning becomes difficult.

\section{B. Our Basic Concept}

To compensate for the influence of time delay, we devised a method to connect the predictor of the PID controller to a conventional PID control system. The predictor predicts the time delays before the output value of the controller. The structure of our basic concept is shown in Fig. 2.

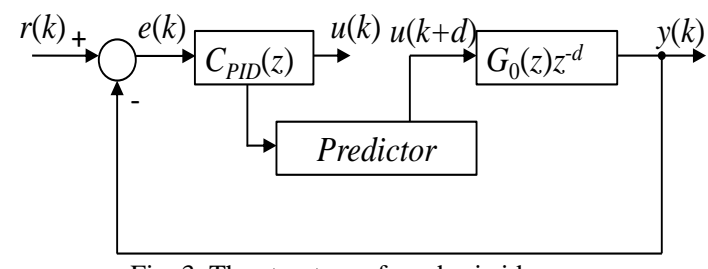

Fig. 3. The structure of our basic idea 
In Fig. 3, $C_{P I D}(z)$ is the discrete PID controller, and $G_{0}(z)$ is the controlled plant without a time delay. If we use the conventional PID method, the controlled variable is given by the following equation.

$$
y(k)=G_{0}(z) z^{-d} u(k)
$$

The proposed method predicts the future output of the PID controller and compensates for the time delay. If the time delay of the controlled plant equals $d$-samples, we predict the future manipulated variable $u(k+d)$. Therefore, the output signal of the proposed method can be written as follows.

$$
y(k)=G_{0}(z) z^{-d} u(k+d)=G_{0}(z) u(k)
$$

Then, the influence of the time delay can be compensated.

\section{PREDICTOR}

\section{A. State Space Model}

To design a predictor, the system needs to be expressed using the state space method. In the state-space method, the system is expressed by a differential equation and the output equation is expressed as Eq. 4.

$$
\left\{\begin{array}{l}
x(k+1)=\boldsymbol{A} x(k)+\boldsymbol{B} u(k) \\
y(k)=\boldsymbol{C} x(k)+\boldsymbol{D} u(k)
\end{array}\right.
$$

In this equation, $x(k), u(k)$ and $y(k)$ denote the state, the input, and output variables of the system respectively. In addition, $A, B, C$ and $D$ denote the state matrix, the input matrix, the output matrix, and the feedthrough matrix respectively. However, in cases where the system model does not have a direct feedthrough, $D$ is the zero matrix.

\section{B. Predictor Design}

A predictor is designed using the state space method. From the Eq.4, the one sample predicted state variable $\tilde{x}(k+1)$ is written as follows.

$$
\tilde{x}(k+1)=\boldsymbol{A} x(k)+\boldsymbol{B} u(k)
$$

Then, we get a two samples predicted state variable by replacing $k$ with $k+1$ in Eq.5 and rewriting as Eq.5.

$$
\begin{aligned}
\tilde{x}(k+2) & =\boldsymbol{A} x(k+1)+\boldsymbol{B} u(k+1) \\
& =\boldsymbol{A}(\boldsymbol{A} x(k)+\boldsymbol{B} u(k))+\boldsymbol{B} u(k+1) \\
& =\boldsymbol{A}^{2} x(k)+\left(\boldsymbol{I}+\boldsymbol{A} z^{-1}\right) \boldsymbol{B} u(k+1)
\end{aligned}
$$

Similarly, we get any number of samples predicted state value by predicting in increments of one sample predicted state variable as follows

$$
\begin{aligned}
\tilde{x}(k+d)= & \boldsymbol{A} \tilde{x}(k+d-1)+\boldsymbol{B} u(k+d-1) \\
= & \boldsymbol{A}^{d} x(k)+\left(\boldsymbol{I}+\boldsymbol{A} z^{-1}+\boldsymbol{A}^{2} z^{-2} \cdots\right. \\
& \left.+\boldsymbol{A}^{d-1} z^{-(d-1)}\right) \boldsymbol{B} u(k+d-1)
\end{aligned}
$$

Then, from Eq. 4 and Eq. 7, the predicted output variable $\tilde{y}(k+d)$ is written as follows.

$$
\begin{aligned}
\tilde{y}(k+d)= & \boldsymbol{C} \tilde{x}(k+d)+\boldsymbol{D} u(k+d) \\
= & \boldsymbol{C} \boldsymbol{A}^{d} \tilde{x}(k)+\left\{\boldsymbol { C } \left(\boldsymbol{I}^{-1}+\boldsymbol{A} z^{-2}+\boldsymbol{A}^{2} z^{-3}+\cdots\right.\right. \\
& \left.\left.+\boldsymbol{A}^{d-1} z^{-d}\right) \boldsymbol{B}+\boldsymbol{D}\right\} u(k+d)
\end{aligned}
$$

\section{PROPOSED METHOD}

\section{A. Discrete Observer for Plants with Time Delay}

The predictor of the controlled plant is connected to the discrete observer. The reason for this is to observe the state variable and make the predictor responsive to the disturbance response. To make the discrete observer for a plant with time delay, we need to connect time delay component to the observer as follows.

$$
\left\{\begin{array}{l}
\tilde{x}(k+1)=\boldsymbol{A} \tilde{x}(k)+\boldsymbol{B} u(k) z^{-\mathrm{d}}+\boldsymbol{K}(y(k)-\tilde{y}(k)) \\
\tilde{y}(k)=\boldsymbol{C} \tilde{x}(k)
\end{array}\right.
$$

In Eq. $12, z^{-d}$ and $\boldsymbol{K}$ denote time delay component and the observer gain respectively. In this way, you make this observer responsive to plants with time delay.

\section{B. The Proposed Method}

We employ the proposed method by connecting the predictors, the time delay component, and a discrete observer, as shown in Fig.4. In this way, we can predict the manipulated value $\tilde{u}(k+d)$ and the control output is given by the following equation.

$$
y(k)=\tilde{u}(k+d) G_{0}(z) z^{-d}=\tilde{u}(k) G_{0}(z)
$$

From Eq. 10, we find that the influence of the time delay is compensated by using the proposed method.

\section{Simulation STUdy}

This section describes the effectiveness of the proposed method using simulation studies. In each case, we use a unit step signal as the reference input signal. The reference input step set point is introduced at $t=0.1[\mathrm{~s}]$, the sampling time $T \mathrm{~s}$ equals $0.001[\mathrm{~s}]$ and the controlled plant is discretized by the zero-order hold method. We also use the signal $r(k+d)$ in Fig. 5 as the reference signal. The simulation result obtained using the proposed method is compared with the results obtained using the Smith predictor and the PID control with time delay compensation [7] as the previous method. 


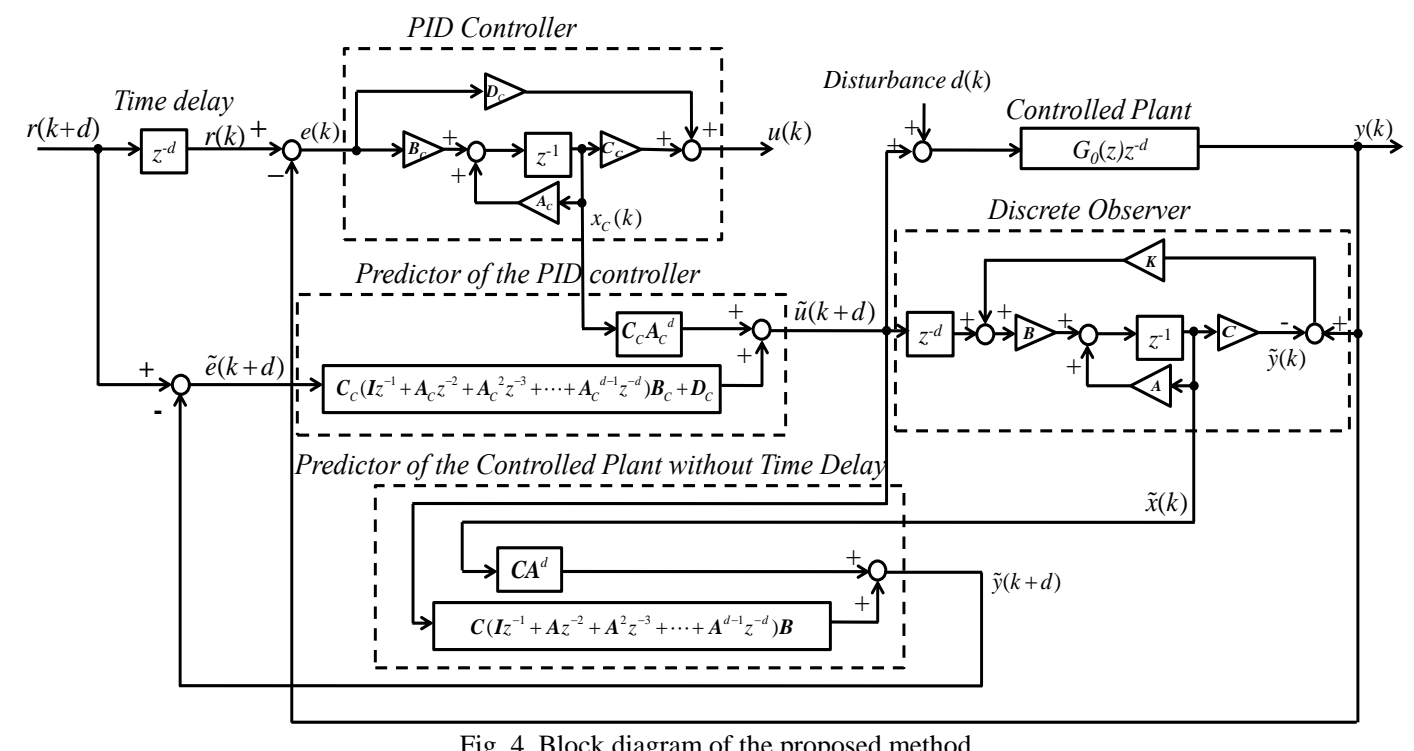

\section{A. Case 1}

In this case, we run a simulation for the controlled plant with a short time delay. To run a simulation, we use the controlled plant expressed as follows.

$$
\begin{aligned}
& G_{0}(s) e^{-L s}=\frac{704}{s(s+49.6)} e^{-0.02 s} \\
& \stackrel{\text { Discretizing }}{\longrightarrow} G_{0}(z) z^{-20}=\frac{0.35 \times 10^{-3} z+0.34 \times 10^{-3}}{z^{2}-1.95 z+0.95 z} z^{-20}
\end{aligned}
$$

The gains of the PID controller $K_{P}, K_{I}$ and $K_{D}$ equal 6, 85 and 0.067 respectively. In addition, the step signal introduced at $t=1.0[\mathrm{~s}]$ and whose amplitude equals -0.2 is applied as the input-side disturbance. The simulation result is shown as (a) in Fig. 5.

\section{B. Case 2}

In this case, we run a simulation for the controlled plant with a longer time delay than in

Case 1. We use the controlled plant given by the following equation.

A predictor is designed using the state-space method. From the equation (3), the one sample predicted state variable $\tilde{x}(k+1)$ is written as follows.

$$
\begin{aligned}
& G_{0}(s) e^{-L s}=\frac{704}{s(s+49.6)} e^{-0.1 s} \\
& \stackrel{\text { Discretzizing }}{\longrightarrow} G_{0}(z) z^{-100}=\frac{0.35 \times 10^{-3} z+0.34 \times 10^{-3}}{z^{2}-1.95 z+0.95 z} z^{-100}
\end{aligned}
$$

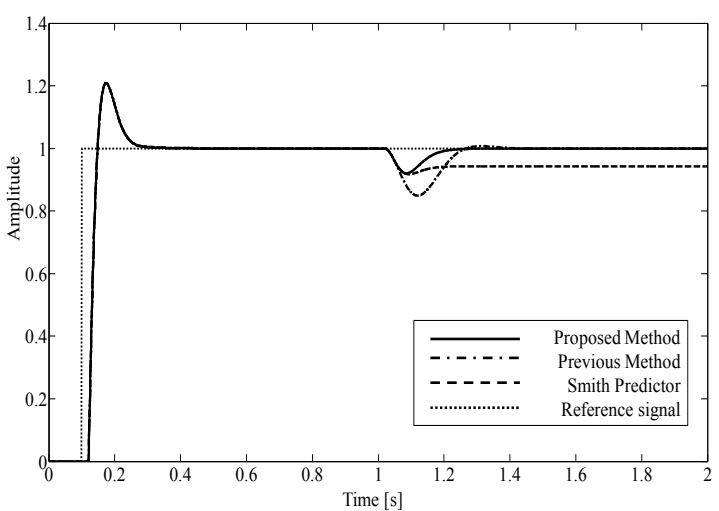

Fig. 5. Simulation result for Case 1
The disturbance and the gains of the PID controller are the same as in Case 1. The simulation result is shown in Fig. 6.

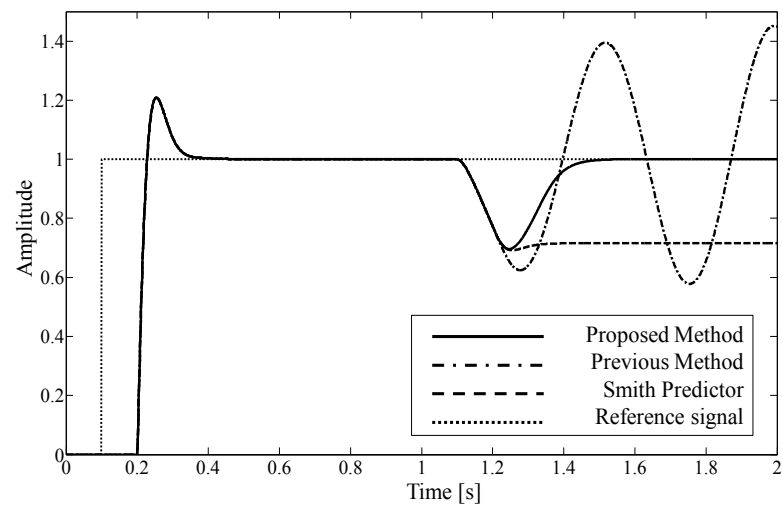

Fig. 6. Simulation result for Case 2

\section{CONCLUSIONS}

In this paper, we have proposed control system to counter the effects of the time delay. For Case 1, the simulation results of the Smith predictor reveal that the steady state error remains under the influence of an input-side disturbance remains. In contrast, the simulation results of the proposed method and the previous method reveal that the steady state error does not remain, and the controlled output signal converges to the reference signal. In addition, it is shown that the fast response of the proposed method is superior to that of the previous method. For Case 2, the disturbance response of the conventional method becomes unstable, while, the simulation result of the proposed method becomes stable. It is shown that the proposed method has positive outcomes in both the input and disturbance responses and it is effective for controlling a plant with a time delay.

\section{REFERENCES}

[1] M. Ohashi, Process Control System, Corona Pub, 1998 (In Japanese)

[2] O. J. Smith "A Controller to Overcome Dead Time," ISA Journal, vol. 6, Feb. 1959, pp. 28-33.

[3] Y. Masukake, M. Ono, H. Ogawa, and Y. Ishida, "Discrete Modified Internal Model Control using PFC for the Plants with a Time Delay," in Proc. ICCAE 2009, Bangkok, March, 2009, pp. 98-102. 
[4] M. Ono, H. Shibasaki, K. Matsumoto, K. Sasaki, and Y. Ishida, "Discrete Modified Smith Predictor Based on Optimal Control Method for a Plant with an Integrator," IEEE International Conference on Systems, Man and Cybernetics (SMC 2010), Istanbul, Oct, 2010, pp. 630-636.

[5] H. Shibasaki, M. Ono, N. Ban, K. Matsumoto, K. Sasaki, and Y.Ishida "Smith Compensator Using Modified IMC for Unstable Plant with Time Delay," ICIEE IPCSIT, vol. 6 LACSIT press, Singapore, 2011, pp. 18-22.

[6] K. Sasaki, M. Ono, N. Ban, K. Matsumoto, and Y. Ishida "A Control System with Time Delay Compensation using PID Predictor," in Proc. ITC-CSCC 2011, Pattaya, July, 2010, pp. 213-216.

[7] T. Furukawa and E. Shimemura, "Predictive Control for Systems with Time Delay,” Int. J. Control, vol. 37, no. 2, 1983, pp. 399-412.

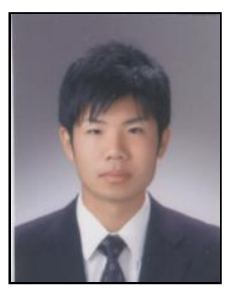

Y. Hikichi was born in Oct. 1988 in Kanagawa, JAPAN. He received the B. E. degree in Electronics and Bioinformatics from Meiji University, Kawasaki, Japan, in 2011. He is currently studying toward the M. E. degree at Graduate School of Science and Technology, Meiji University. His research interests include PID control and its application to linear motor.

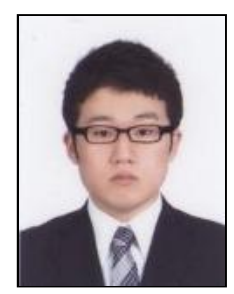

K. Sasaki was born in Nov. 1987 in Kanagawa, JAPAN. He received the B. E. degree in Electronics and Bioinformatics from Meiji University, Kawasaki, Japan, in 2010. He received the M. E. degree in Graduate School of Science and Technology, Meiji University, in 2012. He is currently working toward the company. His research interests include sliding mode control.

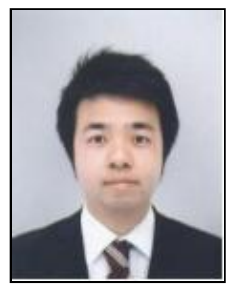

R. Tanaka was born in Oct. 1987 in Fukuoka, JAPAN. He received the B. E. degree in Electronics and Communications from Meiji University, Kawasaki, Japan, in 2010. He received the M. E. degree in Graduate School of Science and Technology, Meiji University, in 2012. He is currently studying toward the Dr. Eng. degree at Graduate School of Science and Technology, Meiji
University. His research interests include Speech Signal Processing, Noise Reduction, Digital Control Systems.

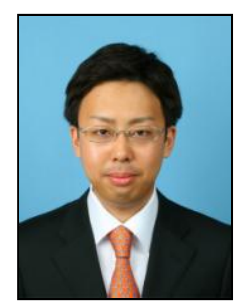

H. Shibasaki was born in Nov. 1988 in Tokyo, JAPAN. He received the B. E. degree in Electronics and Bioinformatics from Meiji University, Kawasaki, Japan, in 2011. He is currently studying toward the M. E. degree at Graduate School of Science and Technology, Meiji University. His research interests include continuous and digital control for a plant with integrator, and an unstable plant and its application to pneumatic equipment. He is a student member of the IEEE, and the IEEJ of Japan.

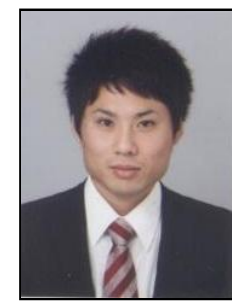

K. Kawaguchi was born in Feb. 1990 in Wakayama, JAPAN. He received the B. E. degree in Electronics and Bioinformatics from Meiji University, Kawasaki, Japan, in 2011. He is currently studying toward the M. E. degree at Graduate School of Science and Technology, Meiji University. His research interests include Dahlin Algorithm for a plant with time delay and its application to pneumatic equipment.

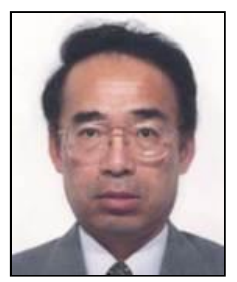

Y. Ishida was born in Feb. 1947 in Tokyo, JAPAN. He received the B. E., M. E., and Dr. Eng. Degrees in Electrical Engineering, Meiji University, Kawasaki, Japan, in 1970, 1972, and 1978, respectively. In 1975 he joined the Department of Electrical Engineering, Meiji University, as a research Assistant and became a Lecturer and an Associate Professor in 1978 and 1981, respectively. $\mathrm{He}$ is currently a Professor at the Department of Electronics and Bioinformatics, Meiji University. His current research interests include signal processing, speech analysis and recognition, and digital control. He is a member of the IEEE, and the IEICE of Japan. 